\title{
UBBER EINIGE UROPTYCHUS-ARTEN DES MUSEUMS ZU KOPENHAGEN
}

\author{
VON \\ Dr. A. J. VAN DAM \\ Zoologisches Laboratorium Amsterdam \\ Mit 5 Abbildungen
}

Die Exemplare sind grossenteils gesammelt von der dänischen Expedition zu den Kei Inseln I921-22 unter Leitung von Dr. Th. Mortensen.

In seiner Reisebeschreibung 1923 gedenkt Herr MORTENSEN ausser seinem Gefährten, H. Jensen, auch den niederländischen Biologen, die ihm und seiner Expedition mit Rat und Tat geholfen haben: M. Weber, H. Boschma, H. C. Siebers, Docters van Leeuwen, K. W. Dammerman, und A. L. J. SuniER, dem heutigen Direktor des jetzt hundertjährigen zoologischen Gartens zu Amsterdam.

Die noch undeterminierten Uroptychus-Arten wurden mir freundlichst von Herrn Dr. K. Stephensen zur Bearbeitung überlassen.

Es zeigte sich, dass drei der vier Arten neu sind: $U$. crassipes, $U$. joloensis, $U$. mortenseni. Die vierte Art ist der schon von der Siboga-Expedition gesammelte $U$..naso vaN DAM.

Ausserdem ist in dieser Arbeit eine ergänzende Beschreibung des, mit den beiden letzten Arten verwandten, $U$ grandirostris Yokoya gegeben, wiewohl dem Museum zu Kopenhagen kein Exemplar dieser Art zugehört.

Dass ich hierzu imstande bin, danke ich der Höflichkeit des Herrn Yokoya, der so freundlich war mir ein seiner Exemplare aus zu leihen zur Vergleichung mit den Siboga-Exemplaren von $U$. naso. Ihm sage ich hier dafür meinen besten Dank.

Weiter stand mir zum Vergleich noch Material aus den Museen Washington D. C., U. S. A. und München zur Verfügung, für dessen Übersendung ich den Herren Dr. W. L. Schmitr und Prof. Dr. H. Balss zu danken habe.

Uroptychus crassipes N. SP.

Stat. 58, Kei Inseln, $5^{\circ} 29^{\prime}$ S., $132^{\circ} 27^{\circ}$ O., $290 \mathrm{~m}$. (Schlamm), I $\sigma^{\prime}$.

Wenn nicht schon ein $U$. granulatus 1903 von BENEDICT beschrieben war und wenn nicht Balss 1913 dieser Art eine neue Variation zugestellt hatte, 
könnte man diese, von der dänischen Expedition gesammelte, Art nicht besser andeuten als mit dem Namen granulatus, denn die Granula, besonders die der Chelipeden, sind sehr auffallend.

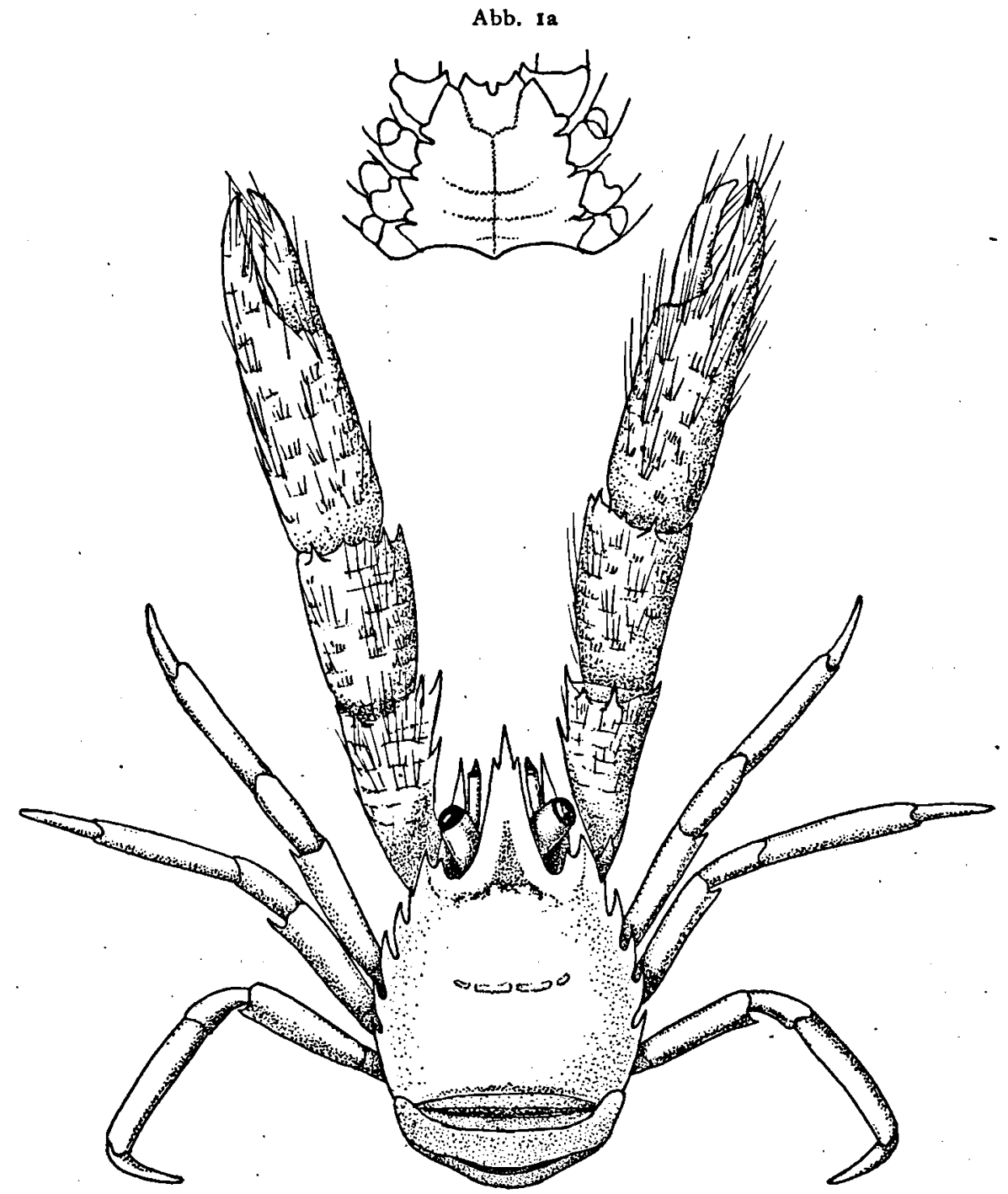

Abb. I. Uroptychus crassipes n. sp. Rückenseite. $\times 6$. Abb. Ia. Sterna von der ventralen Seite gesehen. $\times 6$.

Auch sonst ausser den Granula zeigt $U$. crassipes wohl einige Übereinstimmung mit $U$. granulatus var. japonica BaLss. Beide Arten haben stark verdickte Chelipeden und ein Rostrum, das zwei Seitenspitzen trägt. Die Chelipeden von $U$. crassipes sind aber verhältnismässig dicker und kürzer als von $U$. granu- 
latus var. japonica und die Seitenspitzen seines Rostrums sind bedeutend grösser und deutlicher.

Auch sonst gibt es Unterschiede, die eine Verwechselung völlig ausschliessen. So ist das Längenverhältnis von Rostrum und Rückenschild ein anderes und die Dornen am Seitenrande des Rückenschildes sind bedeutend stärker.

U. crass.: U. gran. var. jap.:

Länge des Rostrums

Länge des Rückenschildes mit Rostrum

Länge der Chelipeden
$3.2 \mathrm{~mm}$

$7.4 \mathrm{~mm}$

I $5.5 \mathrm{~mm}$
$4 \mathrm{~mm}$

I $4.5 \mathrm{~mm}$

$40 \mathrm{~mm}$

Die Oberfläche des Rückenschildes von $U$. crassipes ist von vielen feinen Haaren bedekt und trägt auch mehrere flache Granula, die aber viel weniger ausgeprägt sind als bei $U$. granulatus var. japonica BaLss und ungefähr die Stärke der Granula haben, die das Rückenschild von $U$. granulatus BenEdict zeigt. Eine Regionenbildung ist nicht deutlich, nur ist die Gastralregion gewölbt und vorn gegen das Gebiet des Rostrums deutlich abgegrenzt.

Das Rostrum ist stark und gross, seine Länge ist etwa dreiviertel der Länge des Rückenschildes. Median ist das Rostrum in seiner ganzen Länge tief ausgehöhlt. Das Vorderende ist scharf zugespitzt. Nahe der Spitze zeigt der Seitenrand links und rechts eine gut ausgebildete Nebenspitze. Der Augensulcus ist tief konkav und endet vorn in einen deutlichen Dorn.

Der Anterolateralstachel ist stark. Hinter ihm trägt der Seitenrand des Rückenschildes noch zwei winzige Dörnchen. Gleich hinter der Stelle wo der vordere Sulcus der Cervicalfurche enden würde, steht der erste von vier sehr starken Dornen. Diese vier grossen Dornen des Seitenrandes sind ungefähr gleichförmig und von gleicher Grösse. Der Hintenrand des Rückenschildes ist unbewehrt.

Die Seitenfläche des Rückenschildes trägt ebenfalls Granula. Die Pterygostomialregion endet vorn in einen Dorn.

Der Vorderrand des Sternums der äusseren Maxillipeden trägt eine mediane $U$ förmige Einbuchtung. An den Schenkeln der $U$ ist links und rechts ein winziges Dörnchen zu finden. Vorne endet die Einbuchtung in ein kleines aber deutliches Dörnchen. Links und rechts von diesem Dörnchen biegt der Vorderrand sich nach vorn. An der Grenze von Vorn- und Seitenrand steht ebenfalls ein kleiner Dorn. Weiter zeigt der Seitenrand halbwegs noch eine winzige Spitze und hinten einen langen Dorn.

Der freie Teil des Vorderrandes des Sternums 4 und der vordere Teil des Seitenrandes sind unregelmässig gezähnelt. Dieser gezähnelte Effekt wird noch erhöht durch die Anheftung der Haare, die längs des Randes stehen. Der hintere Teil des Seitenrandes ist rund ausgebuchtet.

Die Sterna 5 bis 7 zeigen ebenfalls einen hinteren rund ausgebuchteten Teil ihres Seitenrandes. Der vordere längere Teil ist etwas mehr seitlich vorgewölbt als beim Sternum 4 . 
Auch die Oberfläche der Sterna zeigt einige wenige flache Granula und mehrere dünne Haare. Übrigens ist sie glatt und glänzend. Die mediane Längsfurche der Sterna 4 bis 7 ist ziemlich tief.

Die Augen reichen bis zur Mitte des Rostrums. Der Stiel ist proximal gleich breit als distal. Die Cornea ist nicht verdickt.

Der Stiel der Antennula ist etwas kürzer als das Rostrum. Sein Vorderende ragt etwa gleich weit vorwärts als die Mitte zwischen der Spitze des Rostrums und dessen Seitenspitze.

Die Schuppe der Antenne ist dorsoventral abgeplattet und vorn scharf zugespitzt. Sie reicht weiter vorwärts als der Stiel und nur sehr wenig weiter als die Seitenspitzen des Rostrums. Der Stiel trägt medio-distal einen Dorn. Die Geissel ist links und rechts abgebrochen.

Die Scherenfüsse sind sehr stark verdickt. Merus, Carpus, Propus und Dactylus tragen sehr grosse schuppenähnliche Granula. Dem Vorderend dieser Granula entspringen viele lange dünne Haare. Das distale Ende der Coxa trägt medio- und latero-ventral einen Dorn. Das Ischium zeigt dorsolateral einen grossen gekrümmten Dorn und distal medio-ventral zwei nebeneinander stehende ziemlich grosse Dornen. Auch das Ischium trägt Granula, die aber viel kleiner sind als bei den mehr distalen Teilen des Scherenfusses. Einige dieser Granula sind ziemlich hoch und spitź so dass sie dörnchenähnlich werden. Eine ganze longitudinale Reihe solcher Dörnchen findet sich medio-ventral am Ischium. Der Merus trägt am distalen Ende mehrere starke Stacheln: ventral je lateral und medial einen, und dorso-medial einen starken Stachel am Vorderende, und hinter diesem einen gleich grossen Dorn am medialen Seitenrande. Dorsal zeigt der Merus noch drei vorwärts gerichtete, schmale aber ziemlich grosse Dornen, welche von der Mitte der drei meist distalen Schuppen entspringen. Auch am distalen Ende des Carpus entspringen dorsal den vorderen Granula schmale, vorwärts gerichtete Dornen. Ventral steht distal je lateral und medial noch ein starker Dorn, diese beiden Dornen finden sich gleich neben einem solchen Schuppendorn. Propus und Dactylus sind unbewehrt, nur trägt der Dactylus an der Innenseite der Schere einen stumpfen Zahn. Der Innenrand beider Finger ist fein gezähnelt. Die Spitzen der Finger sind nach innen gebogen.

Coxa, Merus und Carpus der Pereiopoden 2-4 tragen distal einige starke Dornen. Der Dactylus zeigt ventral eine Längsreihe feiner Zähnchen. Auch die Pereiopoden 2-4 sind stark behaart und tragen schuppenähnliche Granula, die aber viel kleiner sind als die der Scherenfüsse.

Das Abdomen zeigt keine deutliche Granula, wohl ist es stark behaart.

$$
\text { Uroptychus joloensis N. SP. }
$$

Mortensen leg. I9r4. Jolo, 37.8-56.7 m. (Sand, Korallen), I o7.

Das Rückenschild ist länglich, seine Oberfläche ist sehr spärlich besetzt mit langen dünnen Haaren. Übrigens ist die Oberfläche glatt und glänzend, hier 
und da finden sich Grühchen die aussehen wie die Porien der menschlichen Haut:

Der hintere Teil des Seitenrandes hebt sich wie eine deutliche, horizontal ausspringende, Kante von dem übrigen Rückenschilde ab.

Das Rostrum ist schlank. Das Vorderende ist einigermassen dreispitzig aber die Seitenspitzen sind viel weniger ausgeprägt als bei $U$. tridentatus und $U$. crassipes. Die Oberfläche ist konkav, das Rostrum ist nämlich in seiner ganzen Länge median ausgehöhlt. Die Länge des Rostrums ist etwas grösser als die Hälfte der Länge des Rückenschildes.

Der Augensulcus ist tief konkav und endet seitlich in einen winzigen Dorn.

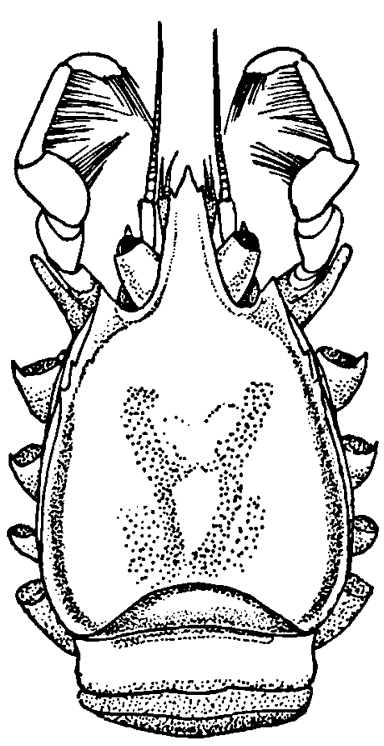

Abb. 2

Der Anterolateralstachel ist gut ausgebildet. Hinter ihm zeigt der Seitenrand des Rückenschildes nur noch zwei deutliche Zähne dicht neben einander. Der vordere steht mehr innenwärts, der hintere steht am Anfang der seitlich vorspringenden Kante des Rückenschildes. Wiewohl der Seitenrand vor und

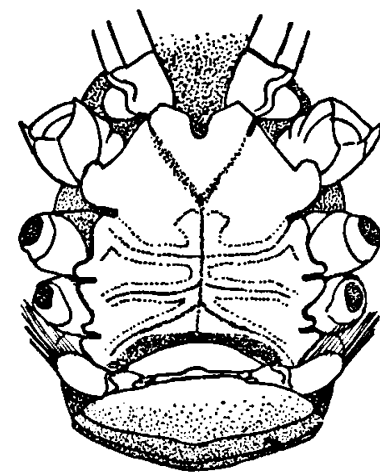

Abb. 2a

Abb. 2. Uroptychus joloensis n. sp. Rückenseite. $X$ Io.

Abb. 2a. Das Tier von der ventralen Seite gesehen. $X$ Io.

hinter diesen beiden Stacheln nicht völlig glatt ist, sind diese sehr kleine Unebenheiten doch kaum nennenswert.

Der Hinterrand des Rückenschildes ist unbewehrt.

Die Branchialregion ist etwas gewölbt und daher deutlich von der Cardialregion abgegrenzt. Die Cervicalfurche ist schwach angedeutet. Die Gastralregion ist nur in ihrem hinteren Teil durch eine untiefe Einsenkung von der Leberregion getrennt.

Unter der Linea anomurica zeigt die Seitenfläche des Rückenschildes drei horizontal deutlich vorspringende Längsleisten von denen die obere gerade unter der Linea anomurica verläuft. Wenn man das Tier von der Rückenseite besieht, sind die mittlere und die untere dieser Längsleisten hier und da seitlich von der dorsalen Oberfläche sichtbar. Die Pterygostomialregion endet vorn in einen Dorn. 
Der Vorderrand des Sternums der äusseren Maxillipeden zeigt eine mediane $U$ förmige Einbuchtung, links und rechts davon eine winzige Spitze. Wo Seitenrand und Vorderrand zusammentreffen steht ein winziges Doppelzähnchen. An der äusseren hinteren Ecke trägt der Seitenrand einen deutlichen Zahn.

Der vordere gerade Teil des Seitenrandes des Sternums der Scherenfüsse

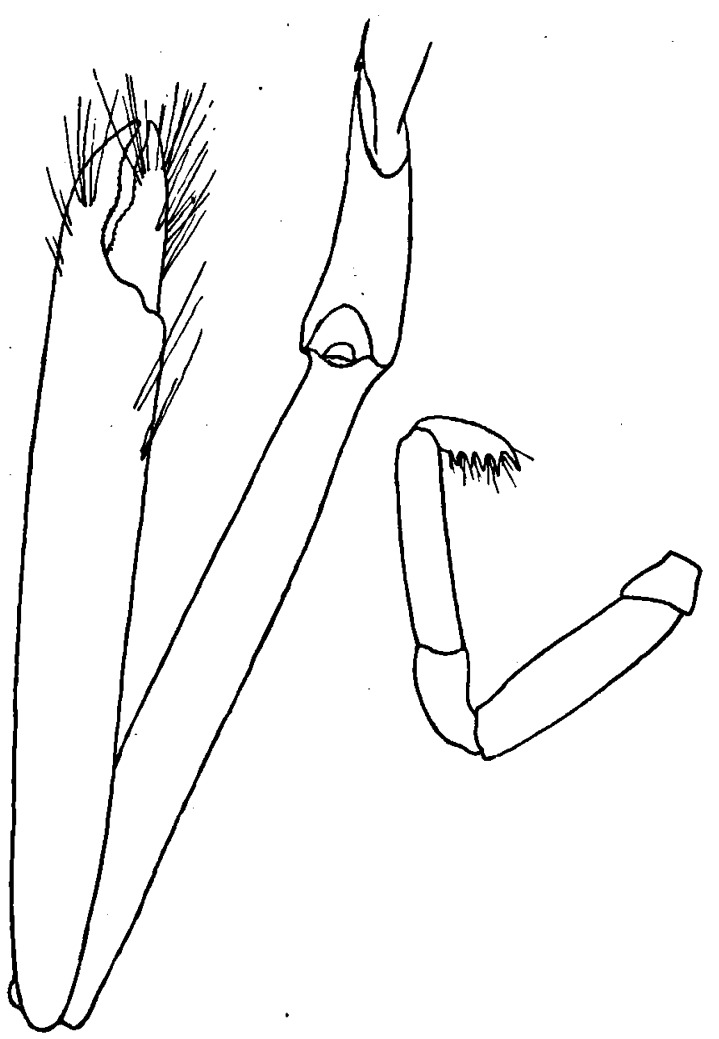

Abb. 2b
Abb. 2c

Abb. 2b. Uroptychus joloensis n. sp. Linker Scherenfuss. $X$ ro.

Abb. 2c. Pereiopode 3. $\times$ Io. ist gezähnelt: links sind drei, rechts zwei deutliche Zähnchen zu sehen. Der hintere Teil des Seitenrandes ist rund ausgebuchtet.

Der Seitenrand der Sterna 5-7 zeigt vorn und hinten eine grosse stumpfe Ausbuchtung. Die mediane Längsfurche der Sterna $4-7$ ist tief. Die ventralen Oberflächen der Sterna 5, 6 und 7 tragen je eine vorspringende Querleiste, die sie fast in der ganzen Breite überquert. Weiter ist die Oberfläche sämtlicher Sterna glatt und glänzend, hier und da steht ein einzelnes Haar.

Auch die Oberfläche des Abdomens ist glatt und glänzend und trägt zerstreut mehrere lange Haare.

Der Augenstiel ist stark, proximal breiter als distal und dieser distale Teil ist wiederum breiter als die Cornea.

Der. Stiel der Antennula ist kürzer als das Rostrum.

Die Schuppe der Antenne ist schmal und reicht ungefähr gleich weit vorwärts als das sie dorsal bedeckende Auge. Der Stiel der Antenne ist länger als die Schuppe aber kürzer als das Rostrum.

Die distalen Glieder der Endopoden der äusseren Maxillipeden sind medial sehr stark behaart.

Die Chelipeden sind lang und schlank. Sie tragen einzelne lange starke Haare, die auf den Fingern zahlreicher sind. Die Coxa trägt medio-ventral einen starken Dorn und dorso-lateral einen noch stärkeren Dorn. Das Ischium trägt lateral zwei starke gekrümmte Dornen, von welchen der vordere der 
grössere ist. Merus und Carpus sind unbewehrt. Die Innenränder der Schere sind fein gezähnelt. Die Spitzen der Finger berühren einander. Am Innenrande der Schere trägt der Dactylus zwei nicht hohe Ausbuchtungen zwischen welchen eine Ausbuchtung des unbeweglichen Fingers hinein passt.

Die Pereiopoden 2-4 sind mit langen starken Haaren besetzt. Der Unterrand ihrer Dactyli trägt mehrere sehr starke Dornen. Der Propus ist unbewehrt.

$$
\text { Masse: }
$$

Länge des Rostums

Länge des Rückenschildes mit Rostrum

Breite des Rückenschildes

Länge des Scherenfusses

$$
\begin{array}{rr}
\text { I,65 mm. } \\
4,25 \mathrm{~mm} . \\
3 & \mathrm{~mm} . \\
2 \mathrm{I} & \mathrm{mm} .
\end{array}
$$

Uroptychus joloensis zeigt durch das dreispitzige Rostrum Ähnlichkeit mit $U$. tridentatus HeNDERSON und mit $U$. crassipes $\mathrm{n}$. $\mathrm{sp}$. Wie aber schon oben gesagt, sind die Seitenspitzen hier viel weniger deutlich ausgebildet. Auch sonst gibt es viele Unterschiede so dass von einer näheren Verwandtschaft mit einer dieser beiden Arten nicht die Rede sein kann.

Auch mit keiner anderen Uroptychus-Art ist eine auffallende Ähnlichkeit fest zu stellen.

Ich möchte an dieser Stelle noch hervorheben, dass auch $U$. xipholepis vaN DAм das Merkmal eines einigermassen dreispitzigen Rostrums zeigt, aber in noch geringerem Masse als es für $U$. joloensis der Fall ist. Die Seitenspitzen sind hier so winzig, dass ich sie in meiner Beschreibung 1933 nicht einmal erwähnt habe. Wohl sind sie aus der Figur 49 zu ersehen.

Auch U. granulatus var. japonica BALSs hat ein dreispitziges Rostrum, wiewohl die Seitenspitzen auch hier nur winzig sind.

Das Vorkommen eines dreispitzigen Rostrums bei allen diesen Arten, die in den übrigen Merkmalen sehr weit auseinander stehen, deutet also nicht auf Verwandtschaft hin, es ist bloss als Konvergenz zu betrachten.

\section{Uroptychus mortenseni N. SP.}

Stat. 52, Kei Inseln, $5^{\circ} 46^{\prime}$ S., I $12^{\circ} 49^{\prime} 35^{\prime \prime}$ O., $35^{2} \mathrm{~m}$ (Schlamm), I o', I mit Eiern.

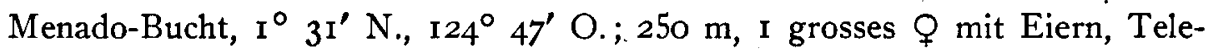
graphenkabel.

Stat. 58, Kei Inseln, $5^{\circ} 29^{\prime}$ S., $132^{\circ} 27^{\prime}$ O., $290 \mathrm{~m}$ (Schlamm), i $\sigma^{\prime}$.

Diese neue Art ist dem $U$. spinimarginatus Henderson in vielen Merkmalen sehr ähnlich.

So zeigt der Seitenrand des Rückenschildes beider Arten eine gleichartige Anordnung der Stacheln: vorn kleinere wenig auffallende Stacheln und dahinten eine Reihe von sehr starken und grossen Dornen. Beide Arten haben ein verhältnismässig langes Rostrum und schlanke Pereiopoden, von denen das $2^{\circ}$ Paar schlanker ist als das $3^{\circ}$ und $4^{\circ}$ Paar. Ausser Unterschieden in mehreren 


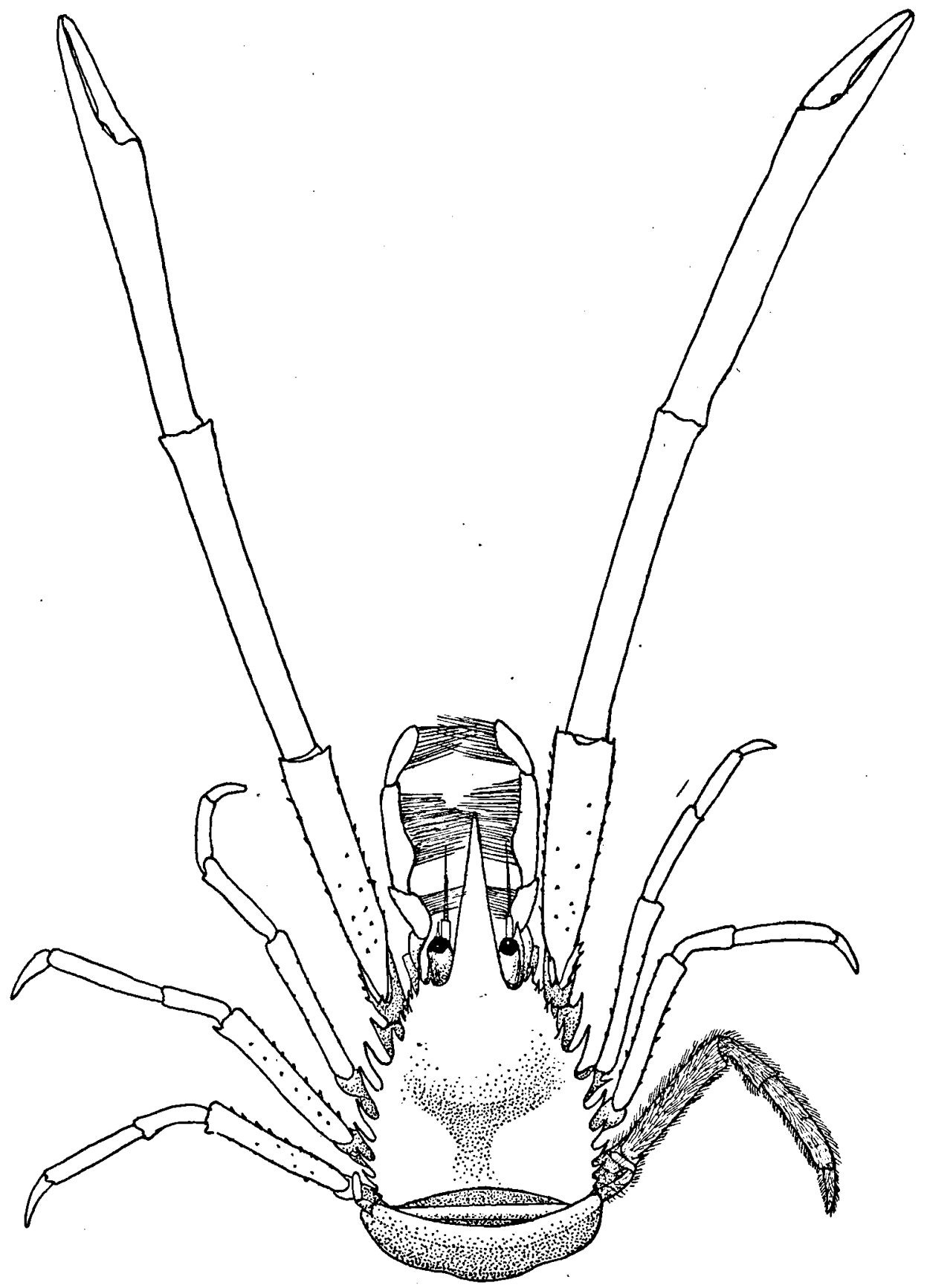

Abb. 3. Uroptychus mortenseni n. sp. Rückenseite. Die starke Behaarung des Tieres ist der Deutlichkeit wegen nur für den rechten Pereiopoden 5 angegeben. $\times 5$. 
Einzelheiten : - Zahl der Dornen des Seitenrandes, und die Reienfolge ihrer Grösse, Länge der Schuppe der Antenne, Grösse des vorderen Pterygostomialstachels, Grössenverhältnis von Länge und Breite des Rückenschildes, grössere Schlankheit der Chelipeden bei $U$. spinimarginatus, und so weiter - ist $U$. mortenseni sofort durch die sehr dichte Behaarung zu erkennen.

Das ganze Tier ist nämlich sehr dicht von feinen, ziemlich kurzen Haaren überdeckt, in die es eingehüllt ist wie in einen Pelz und die selbst den Stacheln am Seitenrande des Rückenschildes und der Schuppe der Antenne nicht fehlen.

Ein zweites auffallendes Merkmal ist die ausserordentlich starke Behaarung von Propus und Dactylus der Endopoden der äusseren Maxillipeden.

Unter seiner dichten Schicht von feinen kurzen Haaren zeigt das Rückenschild eine glatte glänzende Oberfläche. Die Länge des Rückenschildes ist gleich gross oder sehr wenig kleiner als seine Breite.

Der Seitenrand des Rückenschildes ist konvex. Die Gastralregion ist stark gewölbt. Hinter ihr ist der mittlere Teil der Cervicalfurche gut ausgeprägt. Mehr seitlich ist wohl eine Andeutung von seinem vorderen und hinteren Sulcus, diese sind aber durch die dichte Behaarung des Tieres wenig deutlich wahrnehmbar.

Das Rostrum ist lang und stark, es ist etwas kürzer als das Rückenschild. Die Seitenränder sind glattrandig, nur das Weibchen von der Stat. 52 zeigt vorn am Seitenrande einige winzige Unebenheiten, die aber vielleicht nur scheinbar da sind, denn auch das-Rostrum ist sehr dicht von feinen kurzen Haaren eingehüllt und die Ansatzstellen dieser Haare können einen unebenen Rand vortäuschen. Bei dem grossen $\&$ von der Menado-Bucht ist das Rostrum an seiner Basis sehr breit, so dass es die Augen fast völlig überdeckt. In der Dorsalansicht ist seitlich vom Rostrum nur ein Teil der Cornea sichtbar. Der Augensulcus ist mässig tief ausgebuchtet und endet vorn in einen deutlichen Dorn.

Der Anterolateralstachel ist ziemlich lang und stark. Hinter ihm folgen am Seitenrande des Rückenschildes drei kleine Dornen und dann eine Reihe von sieben sehr starken und grossen Dornen, von denen der zweite am allergrössten ist. Hinter diesen grossen Dornen folgen ganz hinten noch ein oder zwei kleine Dornen. Wie

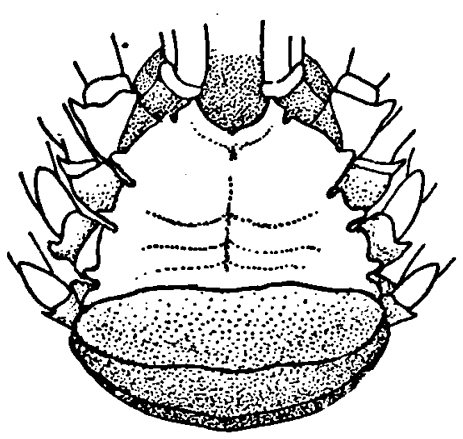

Abb. 3a. Uroptychus mortenseni n. sp. Das Tier von der ventralen Seite gesehen. $\times 5$. schon oben erwähnt, zeigen auch die Dornen des Seitenrandes die für diese Art so charakteristische Behaarung.

Der Hintenrand des Rückenschildes ist unbewehrt.

Die Seitenfläche des Rückenschildes trägt mehrere kleine Dornen, sowohl unter als oben der Linea anomurica. Die Pterygostomialregion endet vorn in einen langen Dorn. 
In seiner Mitte zeigt der Vorderrand der Sterna der äusseren Maxillipeden eine untiefe $\mathrm{V}$ förmige Einbuchtung. Gleich daneben steht links und rechts ein winziges Dörnchen. Seitwärts von diesem biegt der Vorderrand glattrandig und sehr konkav nach vorn. Wenn man die kleine mediane Einbuchtung nicht in Betracht zieht, ist die Form des Vorderrandes einem Halbkreis ähnlich. Der Seitenrand fängt an mit einer winzigen Spitze (hier und da ist es eine Doppelspitze) und hinten trägt er eine nicht hohe Erhöhung.

Die Sterna der Pereiopoden I und 2 haben einen Seitenrand, der vorn einen längeren geraden Teil und hinten einen kurzen rund ausgebuchteten Teil zeigt. Der gerade Teil trägt vorn eine winzige Spitze oder eine kleine Doppelspitze.

Bei den Sterna der Pereiopoden 3 und 4 ist nicht nur der hintere Teil des Seitenrandes sondern auch der vordere einigermassen rund ausgebuchtet.

Auch die Sterna sind behaart.

Die Augen sind kurz, der Stiel ist in seiner ganzen Länge gleich dick. Die Cornea ist nicht breiter als der Stiel. Wie schon oben gesagt, sind die Augen beim grösseren Exemplar von der Menado-Bucht grossenteils vom Rostrum überlagert.

Die Antennula reicht weniger weit vorwärts als das Rostrum. Die beiden Geisseln sind ungefähr gleich lang, die äussere ist sehr viel stärker als die innere.

Der basale Teil der Antenne trägt latero-distal einen Dorn. Die Schuppe ist schlank und endet vorn sehr spitz. Sie reicht um etwa die Hälfte ihrer Länge weiter vorwärts als das Auge. Der Stiel ist wiederum etwas länger als die Schuppe. Die Geissel ist kurz und reicht nicht bis an die Spitze des Rostrums.

Die äusseren Maxillipeden zeigen eine sehr starke Behaarung. Die Haare am Innenrande von Carpus, Propus und Dactylus sind sehr lang. Am Propus und Dactylus sind diese Haare nicht nur sehr lang sondern auch sehr zahlreich, so dass sie einer auffallend starken Bürste ähneln, und der Art ein charakteristisches Gepräge zufügen.

Die Chelipeden sind lang und schlank. Beim grösseren $\sigma$ von der Stat. 58 sind sie ziemlich stark, besonders die linke Schere ist hier dorso-ventral abgeplattet und stark verbreitert. Dem grossen $\&$ von der Menado-Bucht fehlen die Chelipeden. Distal trägt die Coxa dorso-lateral einen grossen starken Dorn, von ungefähr gleicher Grösse als die grossen Dornen am Seitenrande des Rückenschildes. Ein einziges Mal trägt dieser grosse, seitlich zusammengedrückte, Dorn der Coxa zwei dorsal von einander gelegene Spitzen. Ausser einigen winzigen Spitzchen am distalen Rande findet sich noch medio-ventral ein deutlicher Dorn. Dorsal steht am Ischium ein grosser aufwärts gekrümmter Dorn. Medio-ventral trägt das Ischium eine longitudinale Reihe von winzigen Dörnchen, von denen nur der vordere ein wenig grösser ist. Die Oberfläche von Merus und Carpus zeigt ausser einigen grösseren Dörnchen am distalen Gelenk mehrere winzige Dörnchen, die aber wegen der sie überdeckenden Schicht von Haaren sehr wenig auffallen. Diese beiden Glieder sind lang und 
schlank ebenso wie die Schere. Auch die Schere ist von Haaren eingehüllt, sie trägt keine Dornen. Die Innenränder der Schere sind mikroskopisch fein gezähnelt. Der bewegliche Finger trägt eine stumpfe Erhöhung am Innenrande. Die Spitzen beider Finger sind nach innen bebogen. Die Schere ist immer ein wenig dorsoventral zusammengedrückt. Bei der linken Schere des Exemplares von der Stat. 58 ist dies in höherem Masse der Fall, weil die Schere hier stark lateral verbreitert ist. Im Zusammenhang damit sind die Finger hier stark gebogen. Bei geschlossener Schere ist proximal ein ansehnlicher Zwischenraum zwischen den beiden Fingern. Weiter zeigt der bewegliche Finger dieses Exemplars neben dem schon genannten stumpfen Zahn mehr distal noch eine sehr stumpfe Vorwölbung.

Die Pereiopoden 2-4 sind stark behaart und sehr schlank, besonders der Pereiopode 2 ist ausserordentlich schlank. Die Coxa dieser Pereiopoden trägt lateral einen starken Dorn, der Merus zeigt zerstreut einige Dörnchen, die kürzer sind als die sie überdeckenden Haare. Die Dactyli sind gebogen und ihr Unterrand trägt beim dritten und vierten Pereiopoden einige durchsichtige Stacheln, die aber sehr zerbrechlich sind und sich leicht von ihrer Unterlage loslösen.

Das Abdomen ist unbewehrt, es ist gleich stark behaart wie die übrigen Teile des Tieres.

\begin{tabular}{lr}
\multicolumn{2}{c}{ Masse des $\uparrow$ von der Stat. $5^{2}$} \\
Länge des Rostrums & $4.7 \mathrm{~mm}$ \\
Länge des Rückenschildes & $5.5 \mathrm{~mm}$ \\
Breite des Rückenschildes & $5.8 \mathrm{~mm}$ \\
Länge des Merus der Chelipeden & $6.6 \mathrm{~mm}$ \\
Länge des Carpus der Chelipeden & $9.2 \mathrm{~mm}$ \\
Länge der Palma der Chelipeden & $8.4 \mathrm{~mm}$ \\
Länge des Dactylus der Chelipeden & $4.2 \mathrm{~mm}$ \\
\multicolumn{2}{c}{ Masse des $0^{\pi}$ von der Stat. 58} \\
Breite der linken Schere \\
Länge der Palma \\
Länge des Dactylus
\end{tabular}

\section{Uroptychus naso vaN DAM}

Uroptychus naso vaN DAM I933, p. 23, f. $35,36,37$.

Stat. 44, Kei Inseln, $5^{\circ} 39^{\prime}$ S., $132^{\circ} 23^{\prime}$ O., $268 \mathrm{~m}$ (Schlamm) I $\supsetneq$ mit Rhizocephalide.

Stat. 45 , Kei Inseln, $5^{\circ} 48^{\prime} 30^{\prime \prime}$ S., $132^{\circ}$ 1 $4^{\prime}$ O., $270 \mathrm{~m}$ (Sand), I $\bigcirc$ mit Eiern.

Stat. 46 , Kei Inseln, $5^{\circ} 47^{\prime} 20^{\prime \prime}$ S., $132^{\circ}$ I $3^{\prime}$ O., $300 \mathrm{~m}$ (Schlamm), I $ᄋ$ mit Eiern.

Stat. 49, Kei Inseln, $5^{\circ} 37^{\prime}$ Io" S., $13^{\circ} 23^{\prime}$ O., $345 \mathrm{~m}$ (Sand), I $\sigma^{\prime}$. 
Japan, $32^{\circ}$ I $7^{\prime}$ N., $128^{\circ}$ I $1^{\prime}$ O. (Sand), I $\sigma^{\prime}$. Pazifische Expedition des Herm Dr. Th. Mortensen J9I4-I5.

Japan, $32^{\circ}$ Io' N., I $28^{\circ} 20^{\prime}$ O., I $89 \mathrm{~m}$, I $0^{\prime \prime}$, Schönaŭ leg., 1898 . Japan, $32^{\circ} 2^{\prime}$ N., $128^{\circ} 45^{\prime}$ O., $198.45 \mathrm{~m}$, I $\sigma^{\prime}$, Suenson leg., I 898. Japan, $32^{\circ} 25^{\prime}$ N., $128^{\circ} 5^{\prime}$ O., $224.36 \mathrm{~m}$, I $Q$, mit Eiern, Suenson leg., I9r3. Japan, $32^{\circ} 19^{\prime}$ N., I $28^{\circ}$ I $2^{\prime}$ O., $10^{\prime}$, Telegraphenkabel. I 53.09-362.88 m.

Ost Asien, I $\supsetneq$, Telegraphenkabel.

Einige dieser Exemplare zeigen noch welche Farbung. Am dunklen Rückenschild und Abdomen hebt sich ein heller medianer Längsstreifen deutlich hervor.

Von Unterschieden mit den Siboga-Exemplaren ist nur nennenswert, dass bei den meisten Tieren der dänischen Expedition die mediane Längsrinne von Rückenschild und Rostrum gleich stark von Granula überdeckt ist wie die übrigen Teile des Rückenschildes.

\section{Uroptychus grandirostris YOKOYA}

Uroptychus grandirostris Yoкоу 1933, p. 68, f. 29.

Wiewohl diese Art nicht zu der dänischen Kollektion gehört, zeigt sie eine so grosse Übereinstimmung einerseits mit $U$. mortenseni, anderseits mit $U$. naso, dass ich der kurzen Beschreibung ҮокочA's gern einige Bemerkungen zufügen möchte.

Noch immer gilt die Aussprache Yokoya's, dass $U$. grandirostris durch sein grosses Rostrum zu identifizieren ist. Denn es ist die einzige bisjetzt bekannte Art wobei das Rostrum länger ist als das ihm folgende Rückenschild.

In Form und Grösse der Seitenstacheln nähert sich $U$. grandirostris am meisten $U$. spinimarginatus HENDERSON und $U$. mortenseni N. SP. In der Bekleidung seiner Oberfläche stimmt er einigermassen mit $U$. naso überein.

Das Rückenschild macht den Findruck länglich zu sein, wiewohl seine Länge (ohne Rostrum) etwas kleiner ist als die Breite. Die Gastralregion und die Cardialregion sind gewölbt und deutlich von ihrer Umgebung abgegrenzt. Die Cervicalfurche und seine beiden Sulci sind gut ausgeprägt.

Die dorsale Oberfläche von Rostrum und Rückenschild trägt viele kleine Granula, die an vielen Stellen sich bis zu kleinen spitzen Dörnchen erheben. Dies ist besonders der Fall an den seitlichen Teilen des Rostrums, in dem Gebiet zwischen Rostrum und Gastralregion und in der Leberregion. Überdies ist die ganze Oberfläche von kurzen Haaren überdeckt.

Das Rostrum ist sehr stark und lang, länger als das Rückenschild. Die dorsale Oberfläche zeigt in seiner ganzen Länge eine breite und tiefe mediane Rinne. Die Oberfläche dieser Längsfurche ist glatt. Links und rechts von ihr trägt das Rostrum sehr viele kleine dörnchenähnliche Granula. Auch der Seitenrand des Rostrums ist gezähnelt. Der grössere proximale Teil des Rostrums ist gerade und seine dorsale Oberfläche ist horizontal. Vorn ist das Rostrum ein wenig abwärts gebogen so dass die Spitze in einem tieferen Niveau liegt. 


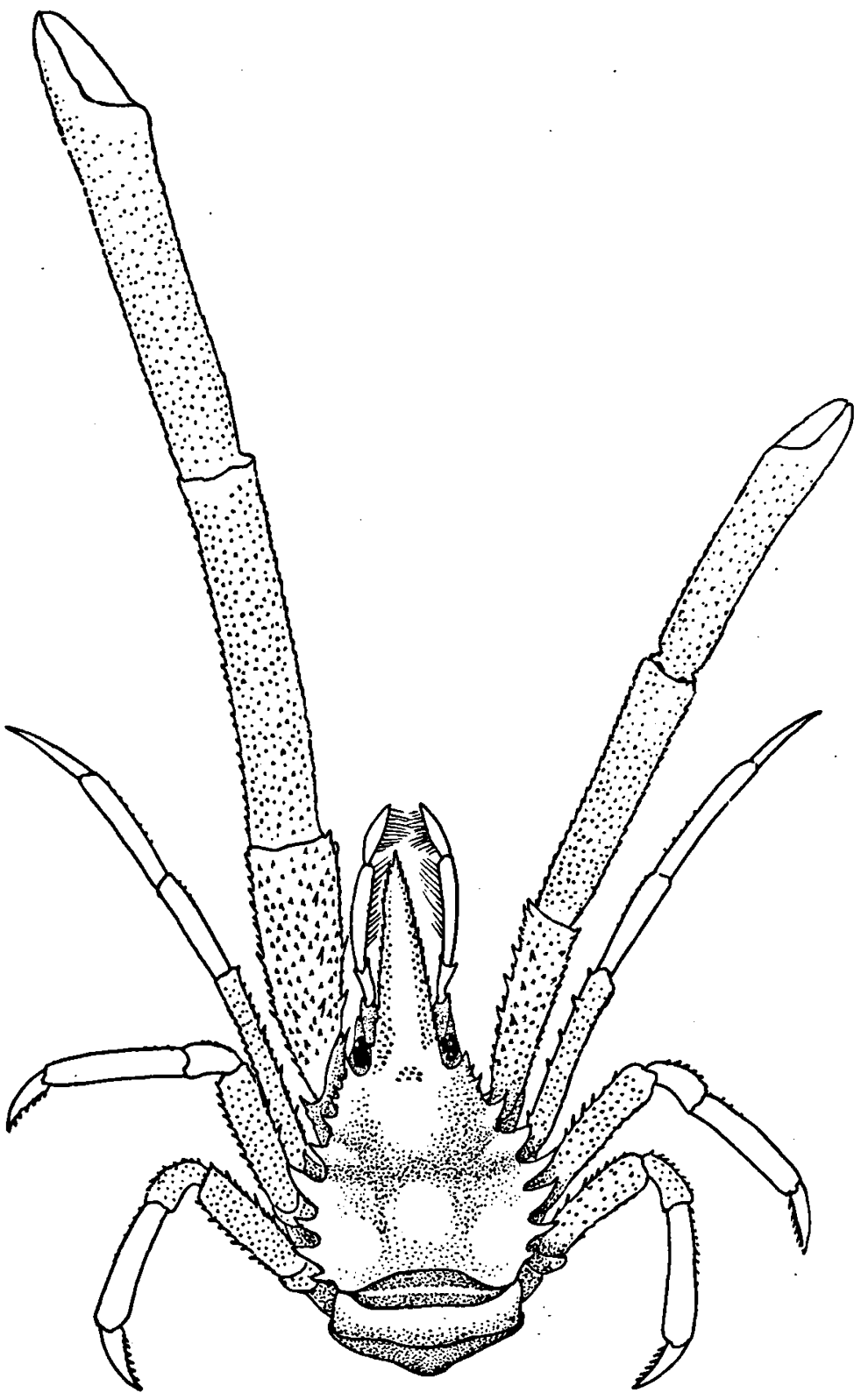

Abb. 4. Uroptychus grandirostris YoKоYA, Rückenseite. $\times 5$.

Der Augensulcus ist nicht besondres tief. Da die Basis des Rostrums sehr breit ist, ist der Verlauf dieses Augensulcus fast mehr dorsoventral als mediolateral. Sein laterales Ende ist nicht deutlich ausgeprägt.

Der Anterolateralstachel ist gut ausgebildet, hinter ihm folgen längs des 
Seitenrandes mehrere kleinere Dörnchen und dann sechs sehr grosse und starke Dornen von denen die mittleren am stärksten sind. Beim Exemplar, das mir Herr Yokoya geschickt hat, ist die Bewaffnung des Seitenrandes ein wenig asymmetrisch. Daher ist hierneben die Abbildung beigefügt eines Weibchens mit Fiern, dessen Photographie Herr Yokоya mir ebenfalls zukommen liess. Auch die Oberfläche der grossen Seitenstacheln trägt kurze Haare. Der Hintenrand des Rückenschildes ist unbewehrt.

Die Seitenfläche des Rückenschildes trägt mehrere Dörnchen. Die Pterygostomialregion endet vorn in einen gut ausgeprägten Stachel, der etwa gleich gross ist wie der Anterolateralstachel.

Das Sternum der äusseren Maxillipeden zeigt an seinem Vorderrande eine tiefe und breite U-förmige mediane Einbuchtung. Links und rechts von den Schenkeln der $\mathrm{U}$ ist die Fortsetzung des Vorderrandes nur wenig gebogen. Der Seitenrand ist besonders in seinem hinteren Teil etwas uneben.

Die Seitenränder der Sterna $4 \rightarrow 7$ haben einen vorde- Abb. 4a. Uroptychus ren geraden Teil, der gezähnelt ist und einen kurzen rund grandirostris YoxoyA. ausgebuchteten hinteren Teil. Das Sternum 4 trägt meh- Von der ventralen Seite rere Granula. Sämmtliche Sterna sind behaart.

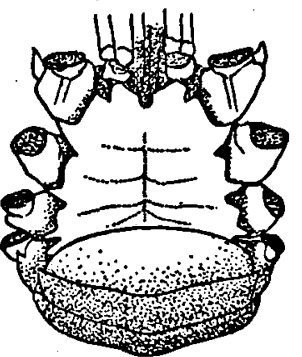

Die Augen sind kurz. Die Cornea ist nicht breiter als der Augenstiel und ungefähr gleich lang wie dieser Stiel.

Der Stiel der Antennula reicht etwa gleich weit vorwärts wie die Hälfte des Rostrums.

Die Antenne ist kürzer als das Rostrum. Ihr Stiel ist etwas kürzer als der Stiel der Antennula. Die Schuppe ist schlank und spitz und ragt etwas weiter vorwärts als das Auge.

Das Exemplar, das mir ausgeliehen wurde hat sehr ungleiche Scherenfüsse. Der linke Scherenfuss ist gross und ziemlich stark. Der rechte Scherenfuss ist viel kürzer und schlanker.

Das von Herm Yokoxa 1933 abgebildete Tier, wie auch die photographische Abbildung, die mir zukam, zeigen beide nur noch den rechten Scherenfuss, von ungefähr gleicher Länge und Stärke als der grössere linke Scherenfuss des hier beschriebenen Tieres. Die Oberfläche beider Scherenfüsse ist von vielen kleinen Dörnchen überdeckt, die nur den Fingern fehlen. Wohl zeigen sich einige wenig auffallende Granula auf den Fingern. Am distalen Ende der Coxa findet sich lateral ein starker, seitlich zusammengedrückter, Stachel. Das Ischium trägt einen grossen, vorwärts gekrümmten dorsalen Dorn, welchem an seinem gebogenen dorsalen Rande noch kleinere Dörnchen aufsitzen können. Am distalem Ende zeigt das Ischium ventral noch einen ziemlich starken Dorn. Der Merus beider Chelipeden trägt drei longitudinale Reihen von starken Dornen, eine ventrale Reihe von nur wenigen Stacheln, eine medioventrale und eine mediodorsale Reihe von vier oder fünf Stacheln. Die Innen- 
fläche der Finger ist fein gezähnelt. Der bewegliche Finger zeigt überdies noch eine nicht hohe stumpfe Vorwölbung, die in eine untiefe Aushöhlung des unbeweglichen Fingers hineinpasst.

Auch die Pereiopoden $2-4$ tragen viele Dörnchen auf ihrer Oberfläche; diese Dörnchen werden an den distalen Teilen granula-ähnlich und fehlen dem

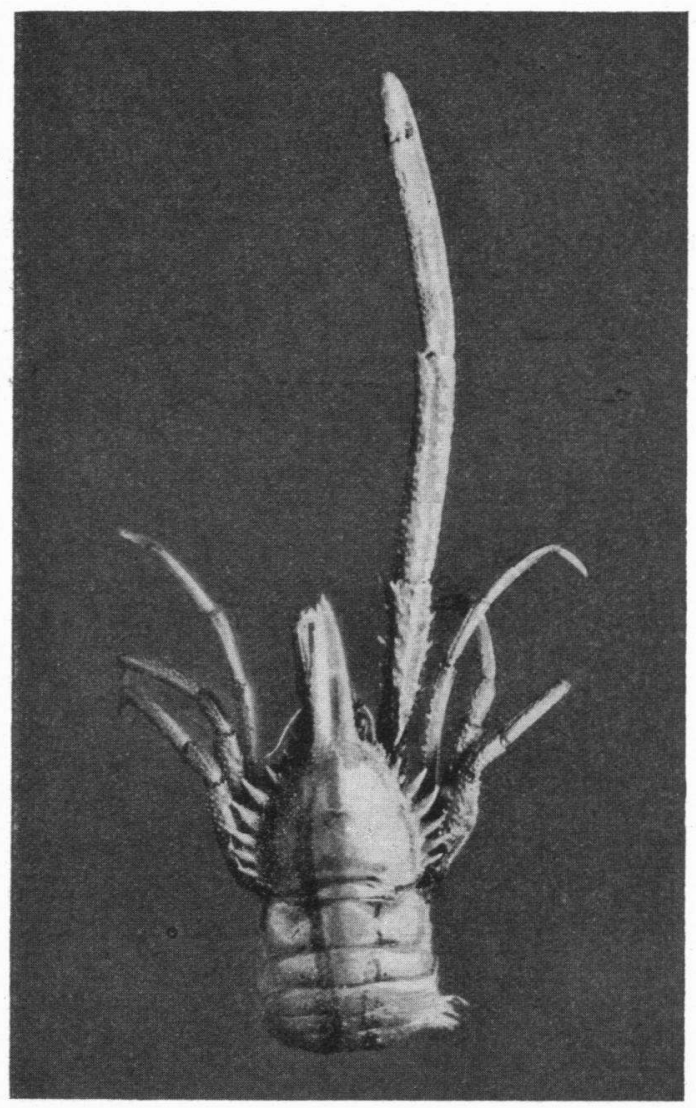

Abb. 5. Photographie von Uroptychus grandirostris YoKOYA.

Dactylus ganz. Die Coxa dieser Schreitfüsse trägt distal lateral und medial einen grossen abgeflachten Dorn. Dem Merus sitzen einige grössere Dornen auf, die, wie bei den Scherenfüssen, in Längsreihen angeordnet sind. Der Carpus des zweiten Schreitfusses ist länglicher als bei den folgenden Pereiopoden. Sein Dactylus ist unbewaffnet. Der Unterrand der Dactyli des dritten und vierten Schreitfusses trägt Stacheln. Sämtliche Schreitfüsse sind spärlich behaart.

Das Abdomen ist unbewaffnet, es ist von kurzen Haaren überdeckt. 
Masse:

$\begin{array}{lr}\text { Länge des Rückenschildes mit Rostrum } & 9.7 \mathrm{~mm} \\ \text { Länge des Rückenschildes ohne Rostrum } & 4.5 \mathrm{~mm} \\ \text { Breite des Rückenschildes } & 4.8 \mathrm{~mm} \\ \text { Länge des linken Scherenfusses } & 30 \mathrm{~mm} \\ \text { Länge des rechten Scherenfusses } & \text { I9 } \mathrm{mm}\end{array}$

\section{LITERATUR}

I 885 Henderson, J. R., Diagnosis of the new Species of Galatheidea collected during the 'Challenger' Expedition. Ann. Mag. Nat. Hist., Londen, (5) I6.

1888 , Report on the Anomura collected by H.M.S. Challenger during the years 1873-1876. Report Sci. Results Voyage H.M.S. Challenger, Zoology 27.

1903 Benedict, J. E., Descriptions of a new genus and forty-six new species of Crustaceans of the Family Galatheidae, with a list of the known marine species. Proc. U.S. Nat. Mus., Washington, 26.

1913 Balss, H., Ostasiatische Decapoden I. Die Galatheiden und Paguriden. Abhandl. Math. Phys. Klasse der K. Bayer. Akad. Wissensch., München, II. Suppl. 9 Abhandlg.

1923 Mortensen, Th., The Danish Expedition to the Kei Islands 1922. Vidensk. Medd. Dansk Naturhist. For. København, 76.

1933 Үокочл, Y., On the distribution of Decapod Crustaceans inhabiting the Continental Shelf around Japan, chiefly based upon the materials collected by S.S. Sóyô-Maru, during the year 1923-1930. Your. College of Agriculture Tokyo 12, Nr. I.

r933 DaM, A. J. vaN, Die Chirostylidae der Siboga-Expedition. Résultats des explorations zoologiques, botaniques, océanographiques et géologiques entreprises aux Indes Néerlandaises orientales en $1899-1900$. Monographie 39, a7, 119. 